\title{
Caenorhabditis elegans killing assay as an infection model to study the role of type III secretion in Burkholderia cenocepacia
}

The Burkholderia cepacia complex (BCC) consists of a group of related genospecies that include important opportunistic pathogens, especially in relation to cystic fibrosis (CF) (Mahenthiralingam et al., 2005). The most common and pathogenically important member of the BCC is Burkholderia cenocepacia, which along with Burkholderia multivorans accounts for the vast majority of BCC isolates from CF patients.

Type III secretion (TTS) systems are implicated in the pathogenicity of a number of Gram-negative bacterial pathogens (Winstanley \& Hart, 2001). Previously we cloned and sequenced the TTS gene cluster from B. cenocepacia ET12 lineage strain J2315 (GenBank AY028431). Furthermore we identified and sequenced TTS gene regions for strains representing genomovar I (B. cepacia) and B. multivorans (strain E243). Whilst genomovar I lacks TTS structural genes, it contains flanking genes, suggesting that a deletion has occurred. $B$. multivorans contains a gene cluster equivalent to that of B. cenocepacia J2315 (Glendinning et al., 2004). It has been demonstrated that a TTS mutant of $B$. cenocepacia $\mathrm{J} 2315$ is attenuated for virulence in a murine model of infection (Tomich et al., 2003), suggesting a role for TTS in pathogenicity. However, the precise role of TTS remains unclear, and the secreted effectors themselves have yet to be identified.

The Caenorhabditis elegans infection model has been used extensively to study bacterial virulence and host-pathogen interactions (Sifri et al., 2005; Alegado et al., 2003). The model has been tested with various members of the BCC (Cardona et al., 2005), but has been used most effectively with B. cenocepacia strain H111 to demonstrate the importance of quorum-sensing (QS) regulation in virulence (Huber et al., 2004). As an alternative to mammalian animal models, we investigated the potential use of a C. elegans killing assay to study the role of TTS in B. cenocepacia. Using the pKNOCK vector system (Alexeyev, 1999), TTS knock-out mutants were constructed in two different strains of B. cenocepacia. A bscs $N$ mutant of B. cenocepacia H111, analogous to the mutant used in a previous study (Tomich et al., 2003), was constructed by cloning an internal region of the $b c s c N$ gene (encoding ATPase) into pKNOCK-Km. The region was PCR-amplified from B. cenocepacia using the oligonucleotide primers 5' -ACCTCTAGAAAGATGATCGACACGCCG-3'

(forward primer containing an $\mathrm{XbaI}$ restriction site) and 5' -ATCCTGCAGCTGGTACTTCGCGATCAAC-3' (reverse primer containing a PstI restriction site), and cloned into pKNOCK-Km plasmid digested with $X b a \mathrm{I}$ and $P s t \mathrm{I}$. In addition, a $b c s c Q R$-virB1 knock-out mutant ( $b c s c Q$ mutant) of B. cenocepacia C5424 (ET12 lineage) was constructed using the pKNOCK-Cm vector (Alexeyev, 1999). A $2.5 \mathrm{~kb}$ SacII-XhoI fragment of the B. cenocepacia $\mathrm{J} 2315$ genome, comprising part of $b c s c Q$, all of $b c s c R$ and part of virB1 (Glendinning et al., 2004), was cloned into pKNOCK-Cm. The pKNOCK-Km-bcscN and $\mathrm{pKNOCK}-\mathrm{Cm}-b \csc Q$ constructs were subsequently introduced into B. cenocepacia H111 and B. cenocepacia C5424, respectively, by electroporation. Cells from $5 \mathrm{ml}$ overnight broth cultures were harvested, washed twice in $10 \%(\mathrm{v} / \mathrm{v})$ glycerol and subsequently resuspended in $0.5 \mathrm{ml}$ of the same solution. Bacterial suspension $(100 \mu \mathrm{l})$ was mixed with DNA, transferred to $0 \cdot 2 \mathrm{~cm}$ electrocuvettes, and subjected to a pulse of $2 \cdot 5 \mathrm{kV}(25 \mu \mathrm{F}$ capacitance and $400 \Omega$ resistance) using a GenePulser II apparatus (Bio-Rad). After electroporation and the addition of $0.9 \mathrm{ml} \mathrm{L}$ broth, cells were incubated at $37^{\circ} \mathrm{C}$ for $2 \mathrm{~h}$ prior to plating onto media containing kanamycin $\left(50 \mu \mathrm{g} \mathrm{ml}^{-1}\right.$; for pKNOCK-Km- $b c s c N$ ) or chloramphenicol (50 $\mu \mathrm{g} \mathrm{ml}^{-1}$; for pKNOCK-Cm-bcscQ). Genomic DNA was isolated from putative mutants using a Wizard Genomic DNA
Purification kit (Promega). The position of the insertion was determined by cloning out the $\mathrm{pKNOCK}-\mathrm{Km} / \mathrm{Cm}$ regions into the $K p n I$ site of pUC19 and sequencing using vector and internal primers. Sequencing confirmed that the pKNOCK construct had inserted into $b c s c N$ and $b c s c Q$ respectively. TTS mutant and wild-type strains were phenotypically similar with respect to growth rate in Luria broth and a series of 20 biochemical tests using the API ZYM kit (bioMérieux).

B. cenocepacia wild-type and two TTS mutant strains were tested in small-scale agar-plate assays for their ability to kill C. elegans, essentially using the method described by Köthe et al. (2003). Bacterial lawns of the test strains and an E. coli control strain were prepared on nematode growth (NG) media to test for infective (slow-killing) effects. Small-scale assays were used in multi-segmented Petri dishes, in which each test was performed in an area of $1.5 \mathrm{~cm}^{2}$. After bacterial growth for $24 \mathrm{~h}$ at $37^{\circ} \mathrm{C}, \mathrm{L} 4$ larvae or adult worms ( $\sim 50$ and $\sim 10$ for the $b c s c Q$ and $b c s c N$ mutants, respectively) were placed on the bacterial lawn. The plates were incubated at $23^{\circ} \mathrm{C}$ and scored for live worms at intervals using a microscope set at $\times 50$ magnification. Results were obtained from three independent experiments (or two for the $b c s c N$ mutant), each experiment testing the ability of the wild-type strain, mutant strain and E. coli OP50 to kill C. elegans.

A comparison of the $b c s c N$ TTS mutant with $B$. cenocepacia $\mathrm{H} 111$ wild-type in the C. elegans slow-killing model of infection at time intervals of 24,48 and $72 \mathrm{~h}$ post-infection indicated a significant difference $(P<0.05)$ at the 48 and $72 \mathrm{~h}$ time-points (Fig. 1a). When the bcscQ mutant was compared with wild-type B. cenocepacia C5424, at time intervals of $6,18,24$ and $42 \mathrm{~h}$, a significant difference $(P<0 \cdot 05)$ was only observed at the $18 \mathrm{~h}$ time-point (Fig. 1b). At the 24 and $72 \mathrm{~h}$ time-points, all worms had been killed by 

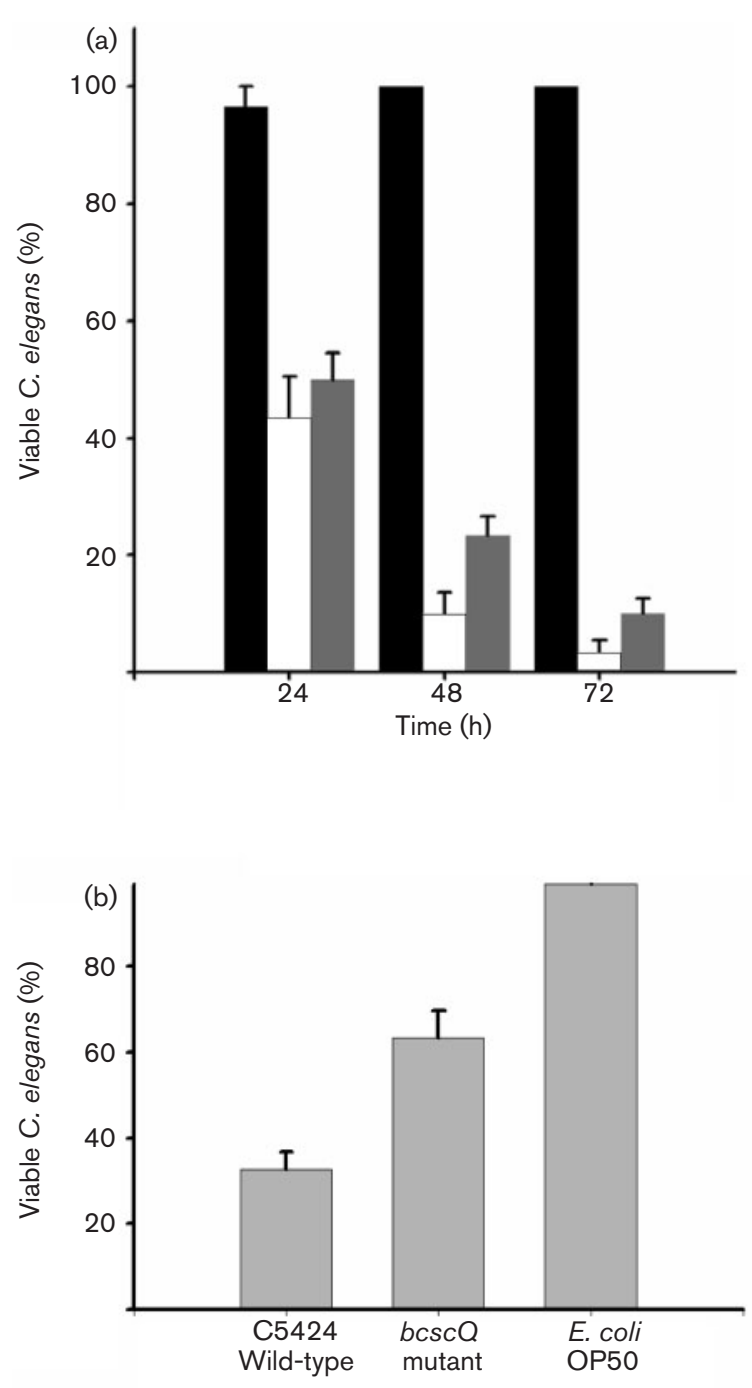

Fig. 1. C. elegans killing assays. (a) C. elegans percentage viability measured at three time-points post-infection; black bars, $E$. coli OP50; white bars, B. cenocepacia $\mathrm{H} 111$; grey bars, $B$. cenocepacia $\mathrm{H} 111-b c s c N$. (b) C. elegans percentage viability measured at the $18 \mathrm{~h}$ time-point.

both wild-type and mutant strains, whereas at the $6 \mathrm{~h}$ time-point, less than $10 \%$ of worms had been killed by either wild-type or mutant. Our findings demonstrate that TTS mutants kill C. elegans more slowly than wild-type strains, but that the effect can only be observed at particular time-points that can vary between $B$. cenocepacia strains. Cardona et al. (2005) have reported significant variation between the C. elegans-killing activities of members of the BCC. Interestingly, strain C5424 was included in the study, but scored as non-pathogenic to C. elegans. Both in our study and the study of Cardona et al. (2005), B. cenocepacia C5424 was obtained as part of an experimental strain panel deemed representative of the BCC (Mahenthiralingam et al., 2000). In our hands, B. cenocepacia C5424 was effective at killing C. elegans. The discrepancy may indicate that one or other version of strain C5424 has mutated.

Slow killing occurs over 2-3 days and involves the accumulation of bacteria in the intestinal lumen (Köthe et al., 2003). Clearly, since mutation did not prevent host infection and killing, factors independent of the TTS system contribute to nematode killing by the BCC. However, our observations suggest that, given the right choice of strain and assay conditions, the $C$. elegans killing assays may provide a more accessible model for the study of defined mutations in the BCC TTS system and should facilitate efforts to resolve the role of this secretion system. C. elegans is well characterized genetically and many mutants are available for study (http://biosci.umn.edu/CGC/ CGChomepage.htm). It has been proposed as a useful model for studying host-pathogen interactions, and especially innate immune responses to infection (Gravato-Nobre \& Hodgkin, 2005). As yet, there are no known conditions in vitro that will trigger the expression of the BCC TTS system genes (Glendinning et al., 2004; Tomich et al., 2003). Studies addressing the interaction between BCC and $C$. elegans may well provide us with a clearer idea of the role of TTS in the pathogenicity of these organisms.

We would like to acknowledge funding from the UK Cystic Fibrosis Trust. In addition, we are grateful to Dr Mikhail Alexeyev (University of South Alabama) for providing the pKNOCK vectors.

Kevin M. Markey, Kerry J. Glendinning, J. Alun W. Morgan, C. Anthony Hart and Craig Winstanley

Division of Medical Microbiology and Genitourinary Medicine, University of Liverpool, Daulby Street, Liverpool L69 3GA, UK

\section{Correspondence: Craig Winstanley (C.Winstanley@liv.ac.uk)}

Alegado, R. A., Campbell, M. C., Chen, W. C., Slutz, S. S. \& Tan, M. W. (2003).

Characterization of mediators of microbial virulence and innate immunity using the Caenorhabditis elegans host-pathogen model. Cell Microbiol 5, 435-444.

Alexeyev, M. F. (1999). The pKNOCK series of broad-host-range mobilizable suicide vectors for gene knockout and targeted DNA insertion into the chromosome of Gram-negative bacteria. Biotechniques 26, 824-828.

Cardona, S. T., Wopperer, J., Eberl, L. \& Valvano, M. A. (2005). Diverse pathogenicity of Burkholderia cepacia complex strains in the Caenorhabditis elegans host model. FEMS Microbiol Lett 250, 97-104.

Glendinning, K. J., Parsons, Y. N., Duangsonk, K., Hales, B. A., Humphreys, D., Hart, C. A. \& Winstanley, C. (2004). Sequence 
divergence in type III secretion gene clusters of the Burkholderia cepacia complex. FEMS Microbiol Lett 235, 229-235.

Gravato-Nobre, M. J. \& Hodgkin, J. (2005). Caenorhabditis elegans as a model for innate immunity to pathogens. Cell Microbiol 7, 741-751.

Huber, B., Feldmann, F., Köthe, M., Vandamme, P., Wopperer, J., Riedel, K. \& Eberl, L. (2004). Identification of a novel virulence factor in Burkholderia cenocepacia H111 required for efficient slow killing of Caenorhabditis elegans. Infect Immun 72, 7220-7230.
Köthe, M., Antl, M., Huber, B., Stoecker, K., Ebrecht, D., Steinmetz, I. \& Eberl, L. (2003). Killing of Caenorhabditis elegans by Burkholderia cepacia is controlled by the cep quorum-sensing system. Cell Microbiol 5, 343-351.

Mahenthiralingam, E., Coenye, T., Chung, J. W. Speert, D. P., Govan, J. R., Taylor, P. \& Vandamme, P. (2000). Diagnostically and experimentally useful panel of strains from the Burkholderia cepacia complex. J Clin Microbiol 38, 910-913.

Mahenthiralingam, E., Urban, T. A. \& Goldberg, J. B. (2005). The multifarious, multireplicon
Burkholderia cepacia complex. Nat Rev Microbiol 3, 144-156.

Sifri, C. D., Begun, J. \& Ausubel, F. M. (2005). The worm has turned - microbial virulence modeled in Caenorhabditis elegans. Trends Microbiol 13, 119-127.

Tomich, M., Griffith, A., Herfst, C. A., Burns, J. L. \& Mohr, C. D. (2003). Attenuated virulence of a Burkholderia cepacia type III secretion mutant in a murine model of infection. Infect Immun 71, 1405-1415.

Winstanley, C. \& Hart, C. A. (2001). Type III secretion systems and pathogenicity islands. J Med Microbiol 50, 116-126. 